\title{
Le développement durable : une influence sur la planification. Du développement régional ?
}

\author{
Michel Laurendeau, CLD de Maria-Chapdelaine \\ Christiane Gagnon, Université du Québec à Chicoutimi ${ }^{1}$
}

1 e développement durable (DD) est à l'ordre du jour des agendas politiques nationaux et internationaux. Le dernier Sommet du G8 en Écosse (juillet 2005), la ratification de l'accord de Kyoto, le Sommet de la Terre à Johannesburg (2002) et, au Québec, le dépôt (novembre 2004) d'un avant projet de loi sur le DD illustre une préoccupation politique face à la survie de la planète, préoccupation confirmée dans les années 1960 par les scientifiques et défendue par les groupes écologistes dans les décennies suivantes. Aujourd'hui, le développement durable n'est plus l'apanage de quelques groupes marginaux, mais oriente les stratégies de plusieurs organisations tant privées, telles les banques et entreprises, que publiques, tels les gouvernements et les institutions du savoir.

C'est la Commission mondiale sur l'environnement et le développement ${ }^{2}$, connue sous le nom de rapport Brundtland, qui a marqué un point tournant dans ce long processus vers un nouveau paradigme de développement. À cet effet, notons le premier Sommet de la terre à Stockholm en 1972. Il y a tout un historique dans le développement du paradigme de DD et celui de la protection de l'environnement. La Commission a défini le DD comme un développement axé sur « la satisfaction des besoins sans toutefois compromettre la capacité des générations futures d'en faire autant ». Par cette définition, elle a introduit la notion d'équité entre les générations et, par extension, d'équité sociale entre le Nord et le Sud, entre les riches et les pauvres, entre les gagnants et les perdants.

Les Sommets de la Terre ont consacré la dimension territoriale du DD, notamment celui de Rio (1992) où il fut question de l'application du DD à l'échelle locale à l'aide de l'Agenda 21 local $^{3}$. Cet outil de planification du DD rejoint le paradigme de développement local viable. Il s'appuie sur une approche de renforcement des micro territoires et des communautés qui considère le local comme un lieu qui façonne le social et l'économique, et vice-versa. Ce retour sur le local, sur les acteurs locaux en tant que sujets intervenants dans toutes les sphères - économique, sociale, culturelle, spatiale - permet un recentrage sur les potentialités de changement contenues dans les pratiques sociales innovatrices ${ }^{4}$. La recherche d'une meilleure qualité de vie, selon des principes d'équité et de solidarité, étant l'objectif du changement et de l'innovation, nous ajoutons le vocable viable qui, selon nous, transcende la notion de durabilité, de pérennité des ressources en vue de la croissance économique.

L'approche territoriale, que Solidarité rurale du Québec revendique pour le $\mathrm{DD}$, considère le milieu de vie comme un système aux interrelations complexes, différent des autres milieux, et engage les communautés dans leur propre développement. Selon eux, le développement ne sera durable que s'il est ancré dans les territoires en respectant les différences et les particularités locales ${ }^{5}$.

Dans un contexte de mondialisation, de délestage du rôle providentiel de l'État et d'un discours de décentralisation/régionalisation en faveur des régions mais surtout des municipalités et des grandes villes, qui s'est notamment traduit au Québec par la nouvelle Loi 170 sur la réorganisation municipale, il est important 
de se demander si le paradigme de DD a influé ou non sur la planification du développement régional (DR) et ce, depuis les années 1980.

Pour répondre à cette interrogation, nous avons choisi la méthode d'une étude de cas longitudinale à trois entrées, à savoir celle de la région du Saguenay-LacSaint-Jean (SLSJ), celle du Centre québécois de développement durable (CQDD, www.cqdd.qc.ca), qui est sis dans la même région, et surtout celle des Sommets économiques qui ont débuté dans les années 1980. Ainsi, à travers deux Sommets économiques (1980, 1986) et deux Planifications stratégiques régionales (1990, 2001), nous tenterons de vérifier l'impact du DD sur l'orientation du discours et des pratiques de DR. L'analyse de ces exercices de planification du DR, sous le chapeau du Conseil régional de concertation et de développement (CRCD), nous permettait ainsi de mesurer l'impact du paradigme de DD sur les pratiques de DR.

Cette réflexion nous mènera vers une meilleure compréhension des relations et des influences entre le DD et le DR et ce, particulièrement en relevant les corrélations et les distorsions du concept à la pratique.

\section{Le Sommet socio-économique de la région du SLSJ (1984) : une première influence des groupes écologistes sur le développement régional ?}

Le Sommet se voulait un exercice permettant l'autodéveloppement de la région par une démarche de concertation, de recherche-action et d'actions. Il se voulait une innovation institutionnelle développée par le gouvernement du Québec afin de favoriser la concertation et de stimuler le développement économique.

«Les participants étaient sélectionnés selon des critères précis et leurs champs d'intérêts devaient être liés aux questions discutées. Ils faisaient partie des décideurs ou d'experts et ils venaient du gouvernement, du patronat, des syndicats, du monde coopératif, des groupes sociaux ou du milieu universitaire. La concertation était conçue comme une démarche organisationnelle cherchant à mobiliser le capital humain par l'implication directe des acteurs dans le processus décisionnel. Le Sommet se voulait une forme de participation à la planification régionale, plus poussée que la simple consultation, plus souple et plus efficace que la planification rationnelle globale ${ }^{6}$. Toutefois, à l'analyse des participants, il appert que la participation des groupes populaires, des petites et moyennes entreprises et du gouvernement fédéral s'est avérée plutôt faible.

Par rapport au DD, alors associé presque exclusivement à la notion d'environnement, il est intéressant de noter le travail fait par les groupes environnementaux pour inclure cette notion dès le départ dans la réflexion et les discussions des leaders régionaux. Déjà en 1981, le Conseil régional de l'environnement (premier conseil régional au Québec) tentait, avec le Conseil régional des loisirs, d'influencer l'orientation strictement économiste du Sommet vers une orientation plus globale de développement. Ceux-ci se sont alors associés avec les groupes populaires afin de modifier la représentativité de la société civile au sein du Sommet. Rappelons que tous ces organismes n'avaient droit qu'à un siège sur 27. Devant ce litige avec l'organisation du Sommet et le CRCD, les groupes populaires (24 au total) prirent la décision de ne pas participer, invoquant que le DR était trop éloigné des préoccupations des groupes populaires.

Toutefois, les groupes écologistes demeurèrent intéressés à poursuivre l'exercice du Sommet, y voyant une occasion unique de faire valoir les conditions d'un autre modèle de développement, du moins d'un autre discours avec lequel le pouvoir devra désormais composer. Quelques mois plus tard, les groupes écologistes acceptaient de travailler à l'élaboration d'une Table sectorielle sur l'environnement ${ }^{7}$. Les écologistes convinrent que cette Table serait composée non pas uniquement d'écologistes, mais inclurait des décisionnels régionaux, dont les représentants des multinationales. Price et Alcan craignaient alors d'être mis au banc des accusés et de devoir justifier les impacts de leurs pratiques industrielles sur l'environnement. Le bloc industriel a réagi vivement à l'option d'écodéveloppement (ancêtre du DD) proposée par la Table, option, selon eux, d'un projet de société utopique et applicable aux pays en voie de développement.

Cette Table ne suscitera pas de débat public. Alors que les écologistes souhaitaient profiter de cette tribune publique pour promouvoir des projets écologiques, d'énoncer les agressions à l'environnement, d'initier les principes de l'écodéveloppement dans une perspective d'éducation du public, la Table a voté le huis clos et le consensus. En outre, le pourcentage pour 
faire passer des propositions de la Table sur l'environnement au Sommet avait été fixé à $75 \%$, ce qui a grandement limité les possibilités d'une discussion élargie à l'échelle régionale. Des 32 propositions déposées à cette Table, 17 ont été fusionnées ou ont subi un rejet avant d'être menées au Sommet.

Ce premier Sommet de 1984, période clef de l'histoire de la concertation régionale, a davantage permis aux écologistes de se faire reconnaître comme des interlocuteurs articulés et incontournables. La table était désormais mise ou préparée afin d'intégrer, éventuellement, la préoccupation environnementale dans le DR.

\section{Le deuxième Sommet socio-économique du SLSJ (1991) : une première tentative d'intégration du DD}

Le Conseil régional de l'environnement a tenu, les 7 , 8 et 9 juin 1990, un colloque sur le DD. Il avait alors été précédé des États généraux de l'environnement (1989) qui faisait notamment un bilan environnemental de la région. À ces deux événements, les décideurs régionaux présents reconnaissent l'importance du DD, compris ici comme la protection de l'environnement, comme une dimension fondamentale de DR. Les participants au colloque décident alors de se doter d'un outil régional pour promouvoir et expérimenter le $\mathrm{DD}$ en région, soit la Région laboratoire du développement durable du SLSJ devenue aujourd'hui le Centre québécois de développement durable (CQDD). Ainsi, la notion de DD, tout comme l'organisme en formation, intégraient le DD au DR comme élément de protection des ressources écologiques. D'ailleurs, la proposition de créer l'organisme Région laboratoire s'est tenue à la Table sectorielle en environnement. « Le projet de région laboratoire peut facilement rejoindre, à moyen terme, l'ensemble des orientations privilégiées par la table sectorielle en environnement. [...] Le but premier de l'implantation de la région laboratoire est de permettre à la population régionale actuelle et future d'améliorer sa qualité de vie en termes de santé, d'emploi, d'éducation et de culture dans le respect des ressources écologiques. [...] Le projet de région laboratoire, par toute son approche multidirectionnelle orientée sur la résolution de problèmes environnementaux, amène comme résultat l'amélioration générale de la qualité de vie ${ }^{8}$. La notion de DD faisait désormais partie du discours officiel sur le DR et était identifié comme le premier des trois principes de base préalables à la démarche de concertation régionale :

1. tenir compte du concept de développement durable et d’aménagement intégré des ressources;

2. miser sur des actions ayant de plus grandes retombées pour la région;

3. favoriser la plus grande prise en main par les acteurs régionaux des leviers de développement.

Toutefois, la compréhension du DD demeure encore limitée à la sphère environnementale (aménagement et gestion des ressources) et n'inclut pas les dimensions sociale et économique. Le DD ne se retrouve exclusivement discuté qu'à la Table sectorielle de l'environnement. L'acceptation des projets aux différentes Tables sectorielles les porte ensuite aux propositions finales du Sommet. Le Sommet est, en quelque sorte, le lieu où sont regroupés les projets des différents secteurs (environnement, agriculture, forêt, mines, coopération, ressources humaines, développement industriel et entrepreneurship, culture, virage technologique, concertation et pouvoir) pour former consensus et engagement des décideurs, sans pour autant qu'il y ait réellement intégration.

\section{Ce premier Sommet de 1984, période clef de l'histoire de la concertation régionale, a davantage permis aux écologistes de se faire reconnaître comme des interlocuteurs articulés et incontournables.}

\section{La Planification stratégique régionale de 1995 : un nouveau regard sur le DR}

La Planification stratégique régionale est la nouvelle formule, proposée par Québec, afin de remplacer les Sommets socio-économiques. Notons à cet effet que la démarche se conclut par une entente-cadre entre la région et le gouvernement du Québec. Elle vise à positionner chaque région par rapport à ses ressources, ses potentiels et ses contraintes en fournissant des balises aux entrepreneurs, aux investisseurs et aux administrateurs publics. C'est une démarche de planification régionale encadrée par un État qui se définit désormais comme un accompagnateur et non comme un État-providence. 
Les intervenants régionaux, sous la direction du CRCD, souhaitaient la participation du milieu et un changement d'attitude envers le développement, celui-ci devant désormais s'appuyer sur la ressource humaine. C'est le CRCD qui était mandataire pour la réalisation de la démarche et avait mis sur pied, pour l'aider dans son processus, un comité aviseur composé de 16 personnes sélectionnées à partir de leurs expertises et connaissances des problématiques régionales. Ce comité a proposé la méthode de travail et l'échéancier de réalisation. Cette méthode présente une démarche qui part d'une synthèse de la problématique régionale qui intègre les facettes économique, sociale et environnementale de la réalité régionale, la recherche de solutions par secteur, l'intégration dans un grand plan d'action, l'appropriation et la réalisation.

La définition de DR proposée tend à se rapprocher de celle du développement durable viable. En effet, le développement serait «le résultat d'un processus de prise en charge conscient, volontaire et structuré qui s'appuie sur des valeurs et des responsabilités individuelles et collectives. Il vise l'amélioration de la qualité de vie des personnes par la mise en place d'infrastructures, de ressources et d'activités répondant de manière durable aux besoins économiques, sociaux, écologiques et culturels des communautés locales et de la collectivité régionale ${ }^{9}$.

Entre 1991 et 1995, l'intégration du DD à la planification du DR ne fait pas partie de l'ensemble des enjeux identifiés.

Des propositions très concrètes font état du DD. Cependant, nous ne retrouvons ces propositions concernant le DD que dans un seul enjeu sur quatre, soit la prise en main de leviers décisionnels sur l'utilisation des ressources naturelles et l'usage prioritaire de nos richesses collectives. Les quatre enjeux se déclinent comme suit :

1. prendre en main les leviers décisionnels sur l'utilisation des ressources naturelles et l'usage prioritaire de nos richesses collectives;

2. doter la région d'une organisation politique et administrative plus efficace;

3. utiliser plus efficacement nos ressources naturelles, humaines, financières et environnementales;
4. positionner la région dans les grands courants économiques, communicationnels, sociaux, culturels et environnementaux mondiaux.

Entre 1991 et 1995, l'intégration du DD à la planification du DR ne fait pas partie de l'ensemble des enjeux identifiés. En ce qui concerne les autres enjeux régionaux, il n'est pas fait mention du DD ou de son influence comme outil de DR. Les intervenants régionaux ont donc adopté une vision du développement qui déborde la stricte dimension économique et qui fait partie de l'ensemble de la démarche, mais, dans la pratique, l'application du DD au plan stratégique régional est encore limitée à l'enjeu des ressources naturelles et de l'environnement.

\section{La Planification stratégique régionale de 2001 : l'affirmation de l'humain comme moteur du développement ?}

L'affirmation de l'importance de l'humain dans le processus du développement n'est pas propre à la région du SLSJ mais fait partie d'un courant mondial, porté notamment par le Programme des Nations Unies sur le développement (PNUD, www.undp.org/french) et popularisé par un indicateur synthèse sur le développement humain. La nouvelle Planification stratégique régionale (PSR) de 2001 n'échappe pas à cette nouvelle tendance. Comme pour l'exercice amorcé en 1995, cette dernière servira de guide dans la détermination des grands axes de DR, tout en validant et en bonifiant le bilan de la dernière PSR.

C'est lors des États généraux sur l'avenir de la région de septembre 1995 (plus de 600 participants) que les partenaires convenaient que l'exercice doit s'appuyer prioritairement sur les ressources humaines. Dans ce sens, l'orientation de développement pour ce plan stratégique de 2001 est « Agir sur nos déterminants en plaçant la personne au centre des préoccupations ${ }^{10}$.

Le principe du DD est réitéré pour guider les partenaires en matière de planification stratégique. Le concept de DD intègre mieux la préoccupation du social, du devenir collectif, des exclus et des minorités. L'être humain semble au centre des stratégies et du discours sur le DR. Par contre, encore une fois, l'analyse des textes produits sur la PSR de 2001 associe directement et clairement le DD au seul secteur des ressources naturelles et de l'environnement. Du point de vue 
de la démarche, il est intéressant de noter que le CRCD a éliminé une étape, soit l'appropriation politique et la consultation populaire sur les résultats du plan stratégique.

Lors de la PSR de 2001, malgré une affirmation de l'importance de l'humain dans le processus du développement, le DD demeure prioritairement associé à l'environnement et aux ressources naturelles. Cela correspond en fait à la définition même adoptée par, la Commission sur l'environnement et le DD du CRCD : «Le développement durable a pour objectif la préservation des systèmes naturels qui nous font vivre : l'atmosphère, l'eau, les sols et les êtres vivants. Il nécessite un rythme de prélèvement des ressources naturelles qui doit respecter la capacité de régénération et d'accroissement naturel et tenir compte de ses effets sur l'ensemble de notre diversité biologique. Il nous faut maintenir et protéger nos espèces végétales et animales qui composent la diversité biologique de notre région $» .{ }^{11}$

\section{DD et DR : du concept à la pratique}

L'adoption du concept de DD, par la communauté internationale et plus récemment par des institutions politiques et économiques, fait suite à une prise de conscience généralisée des problèmes environnementaux et à la pression exercée par de nombreux groupes pendant plusieurs décennies. Toutefois, la dimension territoriale du DD, soit son application à l'échelle régionale et locale, reste encore très fragile, comme le démontre le cas à l'étude. Pourtant, l'analyse des corpus scientifiques tant du côté du développement local, du développement territorial et du développement durable démontre qu'il y a une synergie, voire une parenté entre ces modèles de développement. Toutefois, le travail d'intégration entre le DR et le DD, aussi bien au niveau pratique que théorique, reste à faire. ${ }^{12}$ Malgré tout, l'analyse des exercices de concertation et de planification régionales montre à la fois les avancées mais aussi les limites de cette intégration.

En effet, lors du Sommet de 1984, les écologistes ont compris que la concertation était de mise et qu'il était souhaitable de participer à la démarche de planification régionale pour faire avancer le concept de développement endogène, d'écodéveloppement et de DD. Ainsi, à travers l'analyse des quatre exercices de PSR, il est possible de constater que la dimension environnementale du DD s'est imposée, dès 1991, dans la définition du DR ainsi que dans les principes qui guident les réflexions et les actions de développement. Nous avons aussi vu que le Sommet socioéconomique de 1991 a donné naissance au CQDD, un lieu de promotion du DD, ce qui est une sorte d'innovation sociale.

Par contre, la dimension sociale du DD n'apparaît théoriquement intégrée dans la PSR que lors des deux derniers exercices (1995, 2001). En outre, les stratégies économiques de DD sont en réalité peu présentes, du moins directement ou identifiés comme telles. Le concept de DD ne se retrouve pas documenté ou présent dans la majorité des enjeux, mais uniquement dans les enjeux de type environnement biophysique. Il y a donc une sorte d'incompréhension dans l'interprétation du DD, ce qui entraîne, selon nous, une distorsion entre le DR et le DD, voire une non-intégration. Par ailleurs, il est surprenant de constater que la place accordée au public et aux débats régionaux varie passablement sur près de 20 ans. Le CRCD a accordé, en 1991 et en 1995, un temps, une place aux débats régionaux et à une participation publique, tandis que la PSR de 2001 l'excluait sous prétexte de manque de temps, alors qu'elle introduisait "l'être humain au centre du développement ». Enfin, le CRCD aurait pu prévoir des lieux de consultation, de discussion ou d'animation, même a posteriori, étant donné qu'il a affirmé ce caractère ouvert de son processus dans le temps. C'est pourtant un aspect incontournable du DD.

\section{Conclusion}

En conclusion, cette étude permet de faire une triple constatation sur l'influence du DD dans l'orientation de la planification du DR au SLSJ : 1) l'enjeu environnemental, et notamment la gestion des ressources naturelles, est partie intégrante des exercices de PSR et ce, dès les années 1980; à ce titre, nous pouvons dire que la dimension environnementale a influencé, de façon régulière, la conception de la planification du DR; 2) la dimension sociale est faiblement prise en compte dans la définition des enjeux de DR de même que la participation publique et ce, malgré une affirmation de la place de l'humain dans le développement (2001); 3) l'intégration entre le DR et le DD reste encore à faire pour assurer le devenir des régions.

En analysant le parcours de la dynamique de la planification régionale, nous constatons une dichotomie 
dans la conception du DD, ce qui va à l'encontre même du paradigme qui cherche à intégrer à la fois la durabilité environnementale, la vitalité économique et l'équité sociale, bref la viabilité du développement territorial. L'analyse de contenu illustre cette dichotomie. Globalement, chez un certain nombre de leaders, le DR se résume à l'économie, le développement durable englobe principalement l'environnement, alors que le développement humain est rattaché au social. Il faut dire, à la décharge des acteurs politiques régionaux, que cette dichotomie est présente au sein même du gouvernement du Québec. En effet, le nouveau Plan de DD serait sous la direction unique du ministère de l'Environnement (devenu ministère du Développement durable, de l'Environnement et des Parcs), plutôt que d'une Agence interministérielle regroupant transversalement l'ensemble des acteurs et des sphères du DD.

\section{En analysant le parcours de la dynamique de la planification régionale, nous constatons une dichotomie dans la conception du DD.}

Enfin, nous confirmons qu'il y a eu une influence limitée du DD sur l'orientation de la planification du $\mathrm{DR}$, car le concept de DD a été principalement abordé sous l'angle environnemental. Toutefois, cette intégration reste possible et souhaitable pour l'avenir des régions ressources ou périphériques, à la condition, d'une part, d'interpréter le DD comme un paradigme innovateur et fédérateur et, d'autre part, de reconnaître le rôle essentiel de la société civile et des acteurs territoriaux dans le processus de transformation sociale. Si l'être humain est au cœur des finalités du développement, il est a fortiori l'âme du changement et de son orientation.

\section{Notes et références}

1 Cette publication s'inspire d'un mémoire de maîtrise présenté par M. Michel Laurendeau en 2003 à l'UQAC : Développement durable viable : un changement de pratique pour le développement régional ? Le cas du Centre québécois de développement durable, ainsi que de différents tra- vaux réalisés par Madame Christiane Gagnon professeurechercheure à l'UQAC, directrice de l'axe recomposition des territoires et développement durable du Centre de recherche sur le développement territorial (CRDT). Elle a aussi été la directrice dudit mémoire.

2 Commission mondiale sur l'environnement et la développement (1988), Notre avenir à tous, Montréal, Les Éditions du Fleuve, 454 p.

3 Gagnon, C. (à paraître 2005), « L’Agenda 21 local : un outil de développement durable viable, sous-utilisé par les collectivités territoriales québécoises ", dans Martin Robitaille (dir.), L'Outaouais au carrefour des modèles de développement, Outaouais, UQO.

4 Gagnon, C. (1994), La recomposition des territoires. Développement local viable : récits et pratiques d'acteurs sociaux en région québécoise, Paris, L’Harmattan, 271 p.

5 Solidarité rurale du Québec (2005), Développement durable et approche territoriale : pour une occupation durable des territoires, Nicolet mai, 19 p.

6 Bergeron, G. (1986), La contribution du Sommet sur le développement et l'économie de la région 02 à la mise en place d'un processus d'auto-développement régional, Chicoutimi, GRIR, 36 p.

7 Désy, J., C. Gagnon et P. Prévost (1984), Rapport d'une participation aux tables sectorielles sur l'environnement et le pouvoir régional dans le cadre du Sommet sur l'économie et le développement de la Sagamie, Chicoutimi, GRIR, 32 p.

8 Conseil régional de concertation et de développement, Sommet de la conférence socio-économique régionale Saguenay-Lac-Saint-Jean, 1991. Planification stratégique régionale Saguenay-Lac-Saint-Jean, 1995. Préparatifs pour la PSR 2001, documents de travail, Chicoutimi, 2000. Jonquière.

12 Gagnon, C. (2005), « Développement durable et viable. Enjeux sociaux et scientifiques », dans D. Lafontaine et B. Jean (dir.), Territoires et fonctions. Des politiques aux théories: les modèles de développement régional et de gouvernance en débats, éditions du GRIDEQ et du CRDT, Tome 1, p. 199 à 217. 